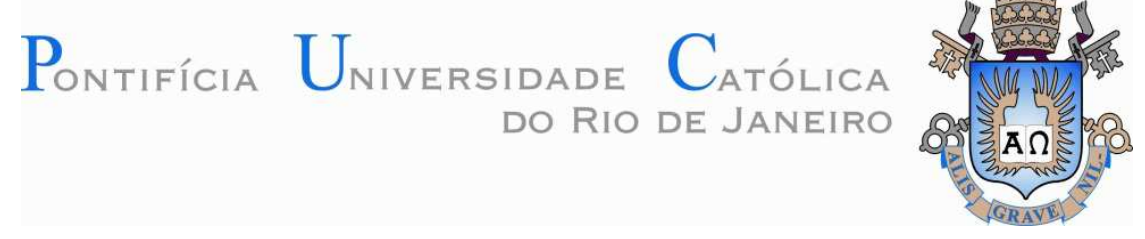

Luiz Gustavo Nery Marques

\title{
Programa de Eficiência Energética Light / ANEEL - Baixa Renda: Experiências da Melhoria dos Processos Logísticos de Troca de Geladeiras
}

Dissertação de Mestrado (opção profissional)

Dissertação apresentada como requisito parcial para obtenção do título de Mestre em Logística pelo Programa de Pós-Graduação em Engenharia Industrial da PUC-Rio.

Orientador: Prof. Nélio Domingues Pizzolato

Rio de Janeiro

Dezembro de 2013 


\section{Pontifícia Universidade Católica $_{\text {at }}$

Luiz Gustavo Nery Marques

\section{Programa de Eficiência Energética Light / ANEEL - Baixa Renda: Experiências da Melhoria dos Processos Logísticos de Troca de Geladeiras}

Dissertação apresentada como requisito parcial para obtenção do título de Mestre em Logística (opção profissional) pelo Programa de Pós-Graduação em Engenharia Industrial da PUC-Rio. Aprovada pela Comissão Examinadora abaixo assinada.

Prof. Nélio Domingues Pizzolato

Orientador

Programa de Pós-Graduação em Engenharia Industrial

Pontifícia Universidade Católica do Rio de Janeiro

Prof. Fernanda Maria Pereira Raupp

Departamento de Engenharia Industrial

Pontifícia Universidade Católica do Rio de Janeiro

Prof. José Eugênio Leal

Programa de Pós-Graduação em Engenharia Industrial

Pontifícia Universidade Católica do Rio de Janeiro

Prof. Jose Eugênio Leal

Coordenador Setorial do Centro Técnico Científico - PUC-Rio

Rio de Janeiro, 04 de dezembro de 2013 
Todos os direitos reservados. É proibida a reprodução total ou parcial do trabalho sem autorização da universidade, da autora e do orientador.

\section{Luiz Gustavo Nery Marques}

Graduado em Engenharia de Produção pela UERJ (Universidade do Estado do Rio de Janeiro) em 2009. Atualmente, é Coordenador de Planejamento na Light Serviços de Eletricidade, atuando no Programa de Eficiência Energética (PEE) voltado a população de Baixa Renda da empresa.

Ficha Catalográfica

Marques, Luiz Gustavo Nery

Programa de Eficiência Energética Light / ANEEL Baixa Renda: experiências da melhoria dos processos logísticos de troca de geladeiras / Luiz Gustavo Nery Marques ; orientador: Nélio Domingues Pizzolato. - 2013.

91 f. : il. (color.) ; $30 \mathrm{~cm}$

Dissertação (mestrado) - Pontifícia Universidade Católica do Rio de Janeiro, Departamento de Engenharia Industrial, 2013.

Inclui bibliografia

1. Engenharia Industrial - Teses. 2. Logística. 3. Gestão de Processos. 4. Programa de Eficiência Energética Baixa Renda. 5. Tarifa Social de Energia Elétrica. I. Pizzolato, Nélio Domingues. II. Pontifícia Universidade Católica do Rio de Janeiro. Departamento de Engenharia Industrial. III. Título. 
Tente mover o mundo: o primeiro passo será mover a si mesmo.

Platão

Who are you?

What are you living for?

Richard Hughes, Tim Rice-Oxley e Tom Chaplin, em trecho de "Perfect Symmetry - Keane"

It's just ten percent luck

Twenty percent skill Fifteen percent concentrated power of will

Five percent pleasure

Fifty percent pain And a hundred percent reason to remember the name Mike Shinoda, em trecho de "Remember The Name - Fort Minor" 


\section{Agradecimentos}

Agradeço aos Professores que tive em toda a trajetória, desde a alfabetização, até o curso que concluo através desse trabalho.

Aos colegas do curso de Mestrado, por promover o aprendizado graças à valiosa troca de experiências e conhecimento.

Ao orientador Professor Nelio Pizzolato, pela disponibilidade em tempo real, sugerindo sempre a melhoria desta dissertação; e ao Professor José Eugenio, por ter sido um colaborador na definição do tema do trabalho em um momento de nebulosidade.

A todos colegas de trabalho da LIGHT, em especial Marliane Mendonça e o amigo André Luis Duarte por colaborarem direta e indiretamente na construção deste trabalho.

A minha mãe Telma e minha sogra Tereza por me motivarem a concluir esse trabalho.

Ao meu pai Jorge Luiz, pelo apoio e incentivo ao estudo durante toda vida.

A minha companheira de todas as horas, Sergiana, por sempre estar a meu lado incondicionalmente.

Por fim, também a todos aqueles que direta ou indiretamente colaboraram para o desenvolvimento do presente trabalho. 


\section{Resumo}

Marques, Luiz Gustavo Nery; Pizzolato, Nélio Domingues. Programa de Eficiência Energética Light / ANEEL - Baixa Renda: Experiências da Melhoria dos Processos Logísticos de Troca de Geladeiras. Rio de Janeiro, 2013. 91p. Dissertação de Mestrado - Departamento de Engenharia Industrial, Pontifícia Universidade Católica do Rio de Janeiro.

O contrato de concessão das empresas de distribuição de energia com a Agência Nacional de Energia Elétrica - ANEEL estabelece que as distribuidoras são obrigadas a investir anualmente um percentual de sua Receita Operacional Líquida em Programas de Eficiência Energética e Pesquisa e Desenvolvimento, de acordo com alguns parâmetros básicos determinados pela Agência. Esses programas têm como foco conscientizar a sociedade para a importância de ações de combate ao consumo irresponsável de energia elétrica, além da viabilidade econômica e estímulo ao desenvolvimento de equipamentos, processos e usos finais de energia mais eficazes. Após a regulamentação da Lei 12.212 de 2010, o perfil do beneficiário foi alterado unicamente para clientes que estão cadastrados na Tarifa Social de Energia Elétrica, com base no Cadastro Único para Programas Sociais do Governo Federal. Esse trabalho busca informar as principais ações de um projeto do Programa de Eficiência Energética direcionadas à população de baixa renda: a seleção dos beneficiários baseada na aplicação de um questionário de prospecção, a distribuição geográfica dos beneficiários, além de demonstrar, através de um exemplo prático e real voltado para a substituição de geladeiras, os impactos provocados na eficiência operacional de uma ação logística, com base em um estudo de acompanhamento dos processos objetivando sua melhoria.

\section{Palavras-chave}

Logística; Gestão de Processos; Programa de Eficiência Energética Baixa Renda; Tarifa Social de Energia Elétrica. 


\section{Abstract}

Marques, Luiz Gustavo Nery; Pizzolato, Nélio Domingues. (Advisor). Energy Efficiency Program - Low Income Light / ANEEL: Improvement Experiences of the Logistics Processes of Replacing refrigerators. Rio de Janeiro, 2013. 91p. MSc. Dissertation - Departamento de Engenharia Industrial, Pontifícia Universidade Católica do Rio de Janeiro.

The concession of power distribution companies with the National Electric Energy Agency - ANEEL establishes that energy distributors are required to invest a percentage of their annual Net Operating Revenue (NOR) in Energy Efficiency Programs (EEP) and Research and Development (P\&D), according to some basic parameters set by the Agency. This program focuses on raising society's awareness over the importance of actions to combat the irresponsible consumption of electricity, in addition to the economic feasibility and to stimulate the development of equipment, processes and more effective end use energy. After the regulation of Law 12.212 / 2010, the profile of the beneficiary was changed to only customers that are registered in the Social Tariff for Electric Power, based in the Single Registry for Social Programmes of the Federal Government. In line with the best management practices applied to various sectors of the world economy, Logistics assumes the responsibility of managing several processes related to the Program of Efficient use of Energy. This master thesis seeks to inform the reader about the main actions of a project of the Program for low-income population, which relying on several steps: selection of the beneficiaries after evaluation of a questionnaire, the geographical distribution of the beneficiaries, and a description of a practical example of substituting old refrigerators. In order to improve the present and similar future processes, the real impacts caused by the operational efficiency of logistics action, are evaluated.

\section{Keywords}

Logistics; Process Management; Low Income Energy Efficiency Program; Social Tariff for Electricity. 


\section{Sumário}

Lista de Figuras

Lista de Tabelas

Lista de Siglas e Abreviaturas

1 Introdução

1.1 Contextualização e justificativa do trabalho 13

1.2 Objetivo do Trabalho 15

$\begin{array}{lll}1.3 & \text { Organização do Trabalho } & 16\end{array}$

2 Referencial Teórico 17

2.1 Logística e Gestão da Cadeia de Suprimentos 17

$\begin{array}{lll}2.1 .1 & \text { Logística } & 17\end{array}$

2.1.2 Gestão da Cadeia de Suprimentos $\quad 21$

2.1.3 Modelo de Lambert e Cooper para a Gestão da Cadeia de Suprimentos

2.1.4 Operador Logístico 27

2.1.5 Terceirização de Serviços 30

2.1.6 Roteirização de Frota 31

2.2 Processos 35

2.2.1 Definições 35

2.2.2 Gestão de Processos 38

3 Mercado de Energia Elétrica - Regras e regulamentações de eficiência energética

4 O Programa de Eficiência Energética (PEE) na Light Serviços de Eletricidade S.A. 
4.1 Programa de Eficiência Energética Light - Baixa Renda Principais Ações

4.1.1 Medição e Verificação - M\&V 48

4.1.2 Ações Educativas 49

4.1.3 Substituição de Equipamentos 53

4.2 Programa de Eficiência Energética Light - Baixa Renda Resultados obtidos e economia estimada 55

4.3 Programa de Eficiência Energética Light - Baixa Renda Comparativo dos resultados obtidos e economia estimada

5 Objeto de estudo - Processo de troca das geladeiras 64

5.1 Descrição do Processo 64

5.2 Avaliação do cenário em seis meses de operação 67

5.3 Identificação de oportunidades de melhoria do processo 69

5.4 Resultados Obtidos 73

5.5 Distribuição Geográfica dos Beneficiários 74

6 Conclusões e recomendações 79

7 Referências bibliográficas $\quad 82$

8 Anexo I: Questionário de Prospecção 88

9 Anexo II: Geladeiras substituídas na cidade do Rio de Janeiro

- distribuição por bairros 


\section{Lista de Figuras}

Figura 1: Evolução da Logística para Gestão da Cadeia de Suprimentos - Adaptado de Ballou (2006)

Figura 2 - Diferenças entre a Logística e a SCM - Adaptado de Ballou (2006)

Figura 3 - Visão da logística integrada - Adaptado de Camelo et al. (2010)

Figura 4 - Framework de Gestão da Cadeia de Suprimentos Adaptado de Lambert e Cooper (2000)

Figura 5 - Classificação das atividades logísticas - Adaptado de Dubke e Pizzolato (2003)

Figura 6 - Principais Avanços da Eficiência Energética no Brasil 42

Figura 7 - Exemplo de Termo de Doação de Geladeira 65

Figura 8 - Exemplo de Boletim de Entregas Diário - BDI 71

Figura 9 - Cidade do Rio de Janeiro ilustrado com os bairros mais atendidos 


\section{Lista de Tabelas}

Tabela 1 - Regras para os investimentos em Programas de Eficiência Energética

Tabela 2 - Legislações e Regulações do Programa de Eficiência Energética

Tabela 3 - Histórico do Programa de Eficiência Energética da Light até o ano de 2007 - Modalidade Baixa Renda

Tabela 4 - Histórico do Programa de Eficiência Energética da Light de 2007 até os dias atuais - Modalidade Baixa Renda

Tabela 5 - PEE 2008-2010 - Economia de energia - Substituição de lâmpadas

Tabela 6 - PEE 2008-2010 - Economia de energia - Doação de sistema de medição

Tabela 7 - PEE 2008-2010 - Economia de energia - Substituição de refrigerador

Tabela 8 - PEE 2011-2013 - Economia estimada - Substituição de lâmpadas

Tabela 9 - PEE 2011-2013 - Economia estimada - Doação de controlador de temperatura de chuveiro

Tabela 10 - PEE 2011-2013 - Economia estimada - Substituição de geladeiras

Tabela 11 - Dados de consumo energético de comunidades atendidas pelo PEE

Tabela 12 - Total de Energia Economizada durante o Ano e o Mês

Tabela 13 - Consumo médio do Bairro Leme

Tabela 14 - Consumo médio do Município de Nilópolis

Tabela 15 - Comparativo de consumo anual estimado

Tabela 16 - Geladeiras entregues entre os meses de abril/2012 e setembro/2012

Tabela 17 - Geladeiras entregues entre os meses de outubro/2012 e abril/2013

Tabela 18 - Distribuição das geladeiras entregues por cidades

Tabela 19 - População das comunidades com UPP da Zona Sul do Rio de Janeiro

Tabela 20 - Maior contingência de geladeiras entregues por bairros da capital 


\section{Lista de Siglas e Abreviaturas}

ANEEL - Agência Nacional de Energia Elétrica

MME - Ministério de Minas e Energia

ROL - Receita Operacional Líquida

PEE - Programas de Eficiência Energética

P\&D - Pesquisa e Desenvolvimento

$M \& V$ - Medição e Verificação

PROPEE - Procedimentos do Programa de Eficiência Energética

MPEE - Manual para elaboração dos Programas de Eficiência Energética

PBE - Programa Brasileiro de Etiquetagem

INMETRO - Instituto Nacional de Metrologia, Qualidade e Tecnologia

PROCEL - Programa Nacional de Conservação de Energia Elétrica

TSEE - Tarifa Social de Energia Elétrica

BPC - Benefício de Prestação Continuada

UPP - Unidades de Polícia Pacificadora 